\title{
Chemodiversity of soil dissolved organic matter
}

4

5 Yang Ding, ${ }^{\dagger}$ Zhenqing Shi, ${ }^{\dagger}, *$ Qianting Ye, ${ }^{\dagger}$ Yuzhen Liang, ${ }^{\dagger}$ Minqin Liu, ${ }^{\dagger}$ Zhi Dang, ${ }^{\dagger}$ Yujun

$8 \dagger$ Key Laboratory of Pollution Control and Ecosystem Restoration in Industry Clusters, Ministry of 9 Education, School of Environment and Energy, South China University of Technology, Guangzhou, 10 Guangdong 510006, People's Republic of China.

$11 \div$ Key Laboratory of Soil Environment and Pollution Remediation, Institute of Soil Science, the Chinese 12 Academy of Sciences, Nanjing 210008, People's Republic of China.

$13 \S$ State Environmental Protection Key Laboratory of Integrated Surface Water-Groundwater Pollution 14 Control, School of the Environmental Science and Engineering, Southern University of Science and

Technology, Shenzhen 518055, People’s Republic of China.

*Corresponding author:

Zhenqing Shi; Tel: +86 20 39380503; e-mail: zqshi@,scut.edu.cn.

20

Number of pages: 14

Number of figures: 10

Number of tables: 3 
27 Table S1. Standardized partial regression coefficients of the multiple stepwise regression between the relative abundance of DOM composition and

28 environmental variables $(\mathrm{n}=33)$.

\begin{tabular}{|c|c|c|c|c|c|c|c|}
\hline Variables & $\begin{array}{c}\text { Aliphatic } \\
\text { compounds }\end{array}$ & $\begin{array}{c}\text { Highly unsaturated and } \\
\text { phenolic compounds }\end{array}$ & $\begin{array}{l}\text { Polyphenolic } \\
\text { compounds }\end{array}$ & $\begin{array}{c}\text { Condensed aromatic } \\
\text { compounds }\end{array}$ & Lipids & $\begin{array}{c}\text { Proteins/amino } \\
\text { sugars }\end{array}$ & Carbohydrates \\
\hline ARI & 0.51 & -0.59 & $\mathrm{~ns}$ & $\mathrm{~ns}$ & ns & 0.69 & 0.66 \\
\hline $\mathrm{pH}$ & ns & ns & ns & ns & ns & ns & ns \\
\hline DOC & -0.23 & ns & ns & $\mathrm{ns}$ & $\mathrm{ns}$ & ns & ns \\
\hline $\mathbf{R}^{2}$ of model & 0.63 & 0.33 & 0.15 & 0.31 & 0.41 & 0.46 & 0.41 \\
\hline$p$ & $<0.05$ & $<0.01$ & $<0.05$ & $<0.01$ & $<0.01$ & $<0.01$ & $<0.01$ \\
\hline
\end{tabular}

ARI, aridity index; DOC, dissolved organic carbon; na, not applicable; ns, not significant. 
Table S2. Partial correlations between environmental variables and aliphatic compounds, highly unsaturated and phenolic compounds, polyphenolic compounds, and condensed aromatic compounds $(p<0.05)$.

\begin{tabular}{ccccc}
\hline & \multicolumn{3}{c}{ Tested variable } \\
\cline { 2 - 5 } Controlled variable & ARI & Clay & $\mathrm{pH}$ & DOC \\
\hline Aliphatic compounds & & & & \\
ARI & na & 0.52 & ns & ns \\
Clay & 0.53 & na & ns & ns \\
pH & 0.53 & 0.54 & na & na
\end{tabular}

Highly unsaturated and phenolic compounds

$\begin{array}{ccccc}\text { ARI } & \text { na } & \text { ns } & \text { ns } & \text { ns } \\ \text { Clay } & -0.47 & \text { na } & \text { ns } & \text { ns } \\ \text { pH } & -0.47 & \text { ns } & \text { na } & \text { ns } \\ \text { DOC } & -0.59 & -0.47 & 0.41 & \text { na }\end{array}$

\section{Polyphenolic compounds}

$\begin{array}{ccccc}\text { ARI } & \text { na } & \text { ns } & \text { ns } & 0.42 \\ \text { Clay } & \text { ns } & \text { na } & \text { ns } & \text { ns } \\ \mathrm{pH} & \mathrm{ns} & -0.38 & \text { na } & 0.39 \\ \text { DOC } & -0.36 & -0.41 & \text { ns } & \text { na }\end{array}$

\section{Condensed aromatic compounds}

$\begin{array}{ccccc}\text { ARI } & \text { na } & -0.44 & \text { ns } & 0.38 \\ \text { Clay } & \text { ns } & \text { na } & \text { ns } & \text { ns } \\ \text { pH } & \text { ns } & -0.42 & \text { na } & 0.36 \\ \text { DOC } & -0.50 & -0.57 & 0.51 & \text { na }\end{array}$


Table S3. Partial correlations between environmental variables and lipids, proteins/amino sugars, and

34 carbohydrates $(p<0.05)$.

\begin{tabular}{|c|c|c|c|c|}
\hline \multirow{2}{*}{ Controlled variable } & \multicolumn{4}{|c|}{ Tested variable } \\
\hline & ARI & Clay & $\mathrm{pH}$ & DOC \\
\hline \multicolumn{5}{|l|}{ Lipids } \\
\hline ARI & na & 0.61 & $\mathrm{~ns}$ & ns \\
\hline Clay & $\mathrm{ns}$ & na & ns & ns \\
\hline $\mathrm{pH}$ & $\mathrm{ns}$ & 0.63 & na & $\mathrm{ns}$ \\
\hline DOC & $\mathrm{ns}$ & 0.65 & ns & na \\
\hline \multicolumn{5}{|l|}{ Proteins/amino sugars } \\
\hline ARI & na & $\mathrm{ns}$ & ns & $\mathrm{ns}$ \\
\hline Clay & 0.58 & na & ns & ns \\
\hline $\mathrm{pH}$ & 0.56 & $\mathrm{~ns}$ & na & ns \\
\hline DOC & 0.72 & 0.47 & -0.50 & na \\
\hline \multicolumn{5}{|l|}{ Carbohydrates } \\
\hline ARI & na & ns & ns & $\mathrm{ns}$ \\
\hline Clay & 0.58 & na & -0.43 & $\mathrm{~ns}$ \\
\hline $\mathrm{pH}$ & 0.46 & ns & na & $\mathrm{ns}$ \\
\hline DOC & 0.66 & 0.39 & -0.55 & na \\
\hline
\end{tabular}

35

ARI, aridity index; DOC, dissolved organic carbon; na, not applicable; ns, not significant. 

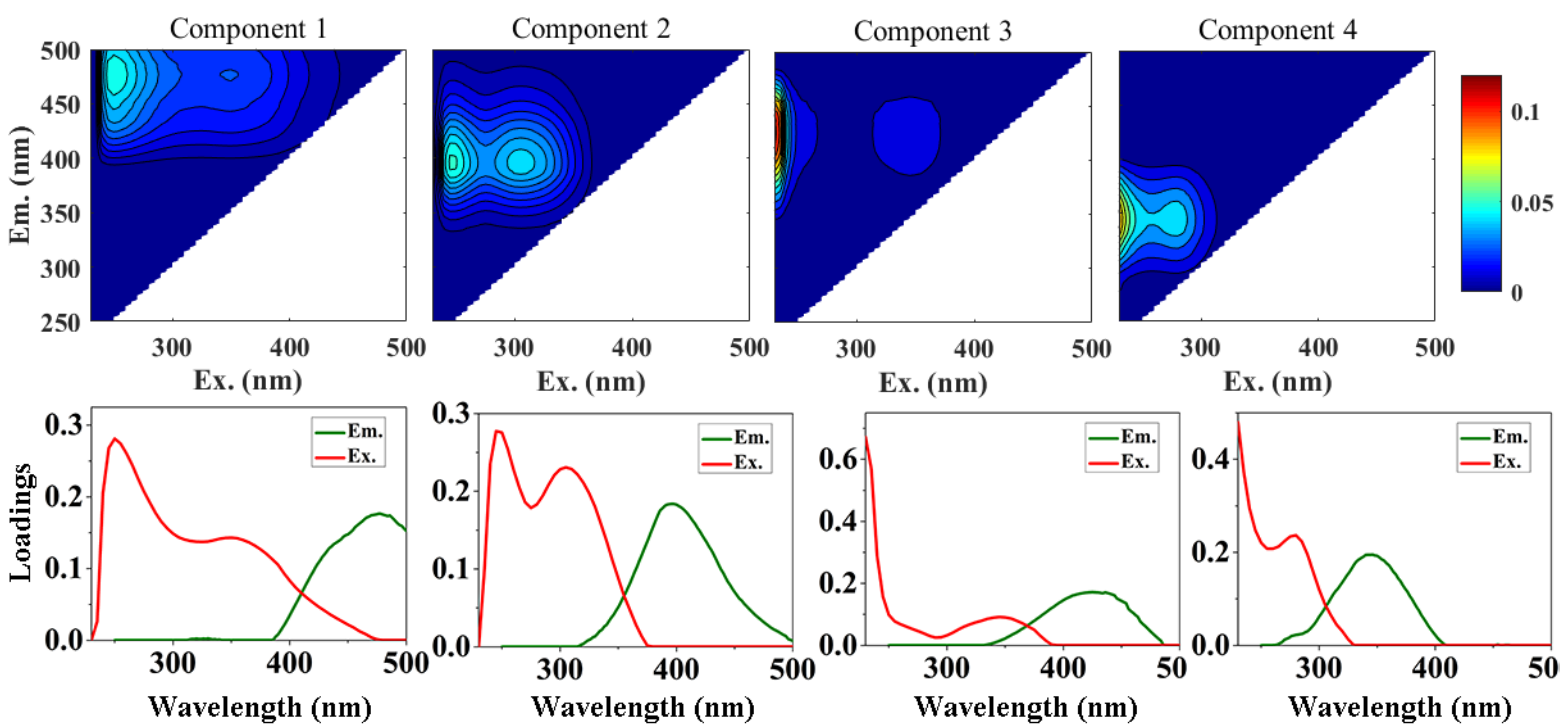

Ex. (nm)

Wavelength (nm)

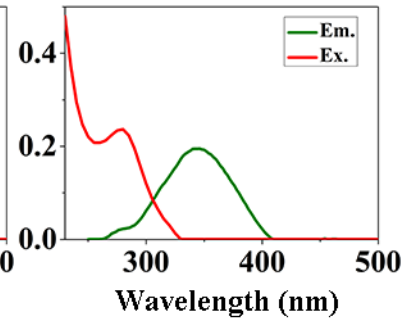

Figure S1. 3D-EEM spectra and loadings of the fluorescence components identified using PARAFAC in 33 DOM samples. Four fluorescence components were identified, including three humic-like components (components 1-3) and one protein-like component (component 4). 
(a)
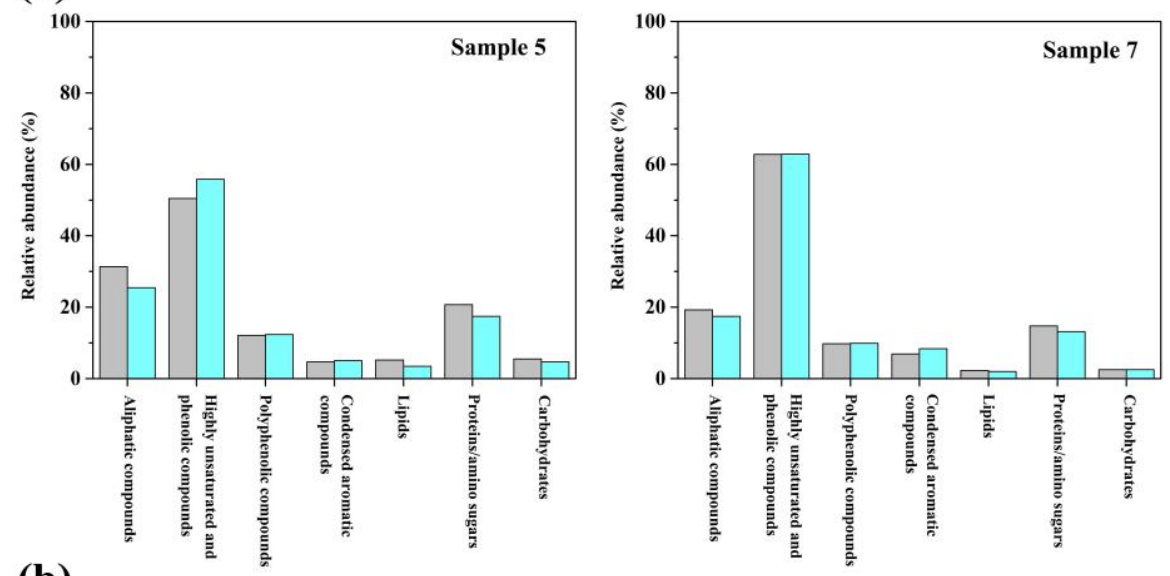

(b)
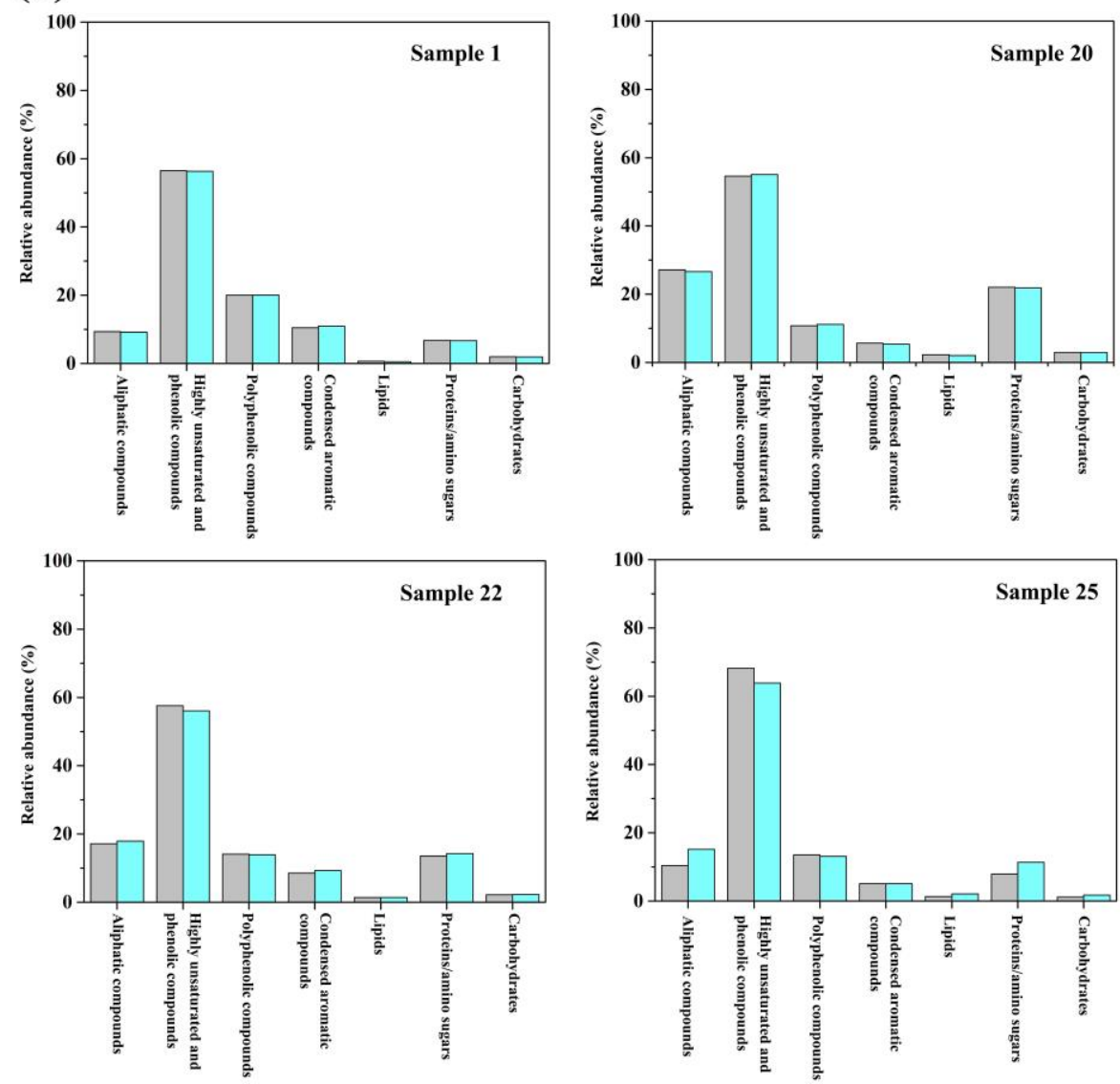

Figure S2. Comparison of the relative abundance of major organic compounds in (a) DOM samples extracted from soils with or without air-drying (i.e. samples 5 and 7), and (b) duplicates of four soil DOM samples (i.e. samples 1, 20, 22 and 25). Major DOM compounds included aliphatic compounds, highly unsaturated and phenolic compounds, polyphenolic compounds, condensed aromatic compounds, lipids, proteins/amino sugars, and carbohydrates. 


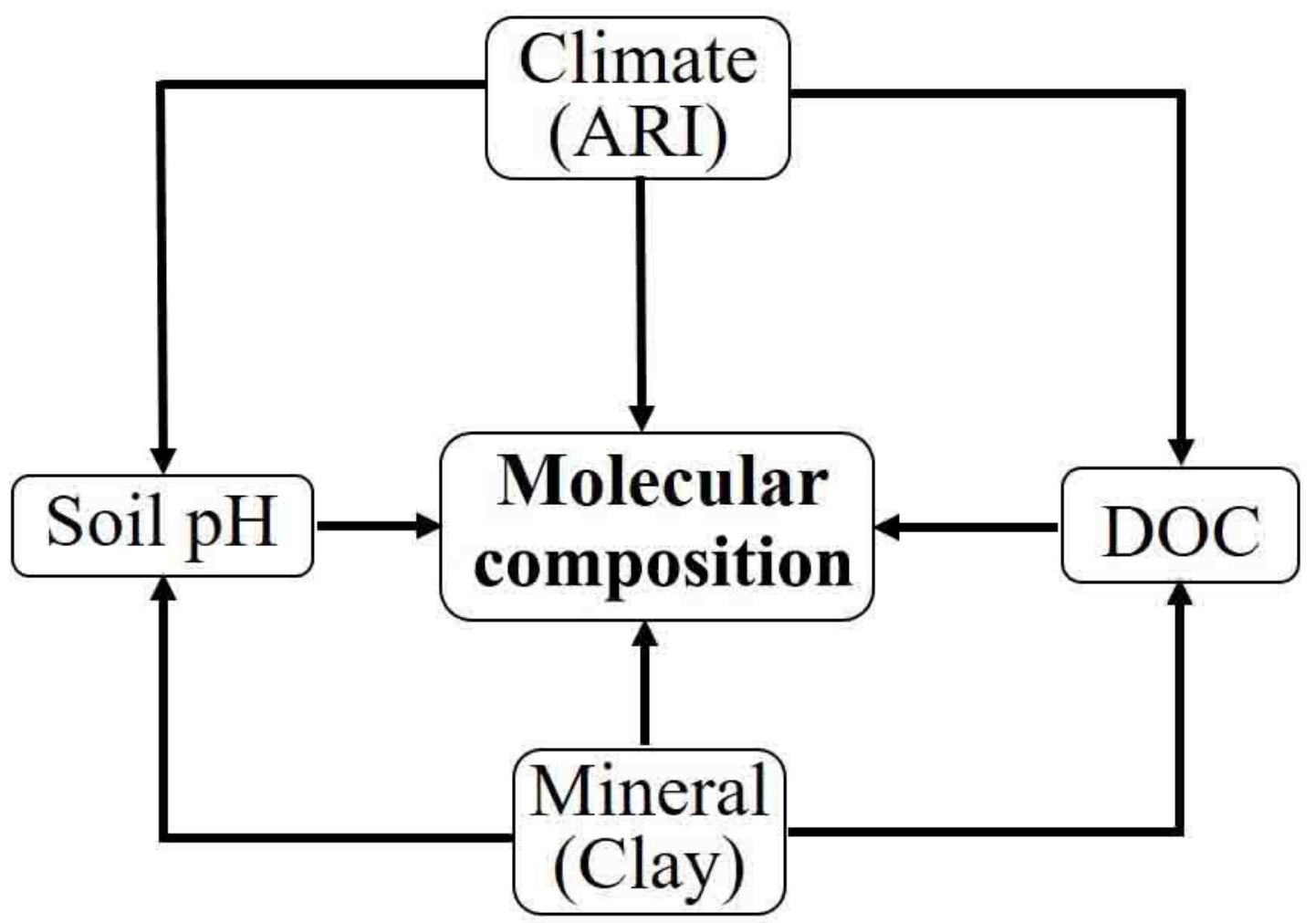

48 Figure S3. A priori model for the structural equation modeling analysis of DOM molecular composition 49 including aliphatic compounds, lipids, proteins/amino sugars, carbohydrates, highly unsaturated and 50 phenolic compounds, polyphenolic compounds, and condensed aromatic compounds. ARI, aridity index; 51 DOC, dissolved organic carbon. 

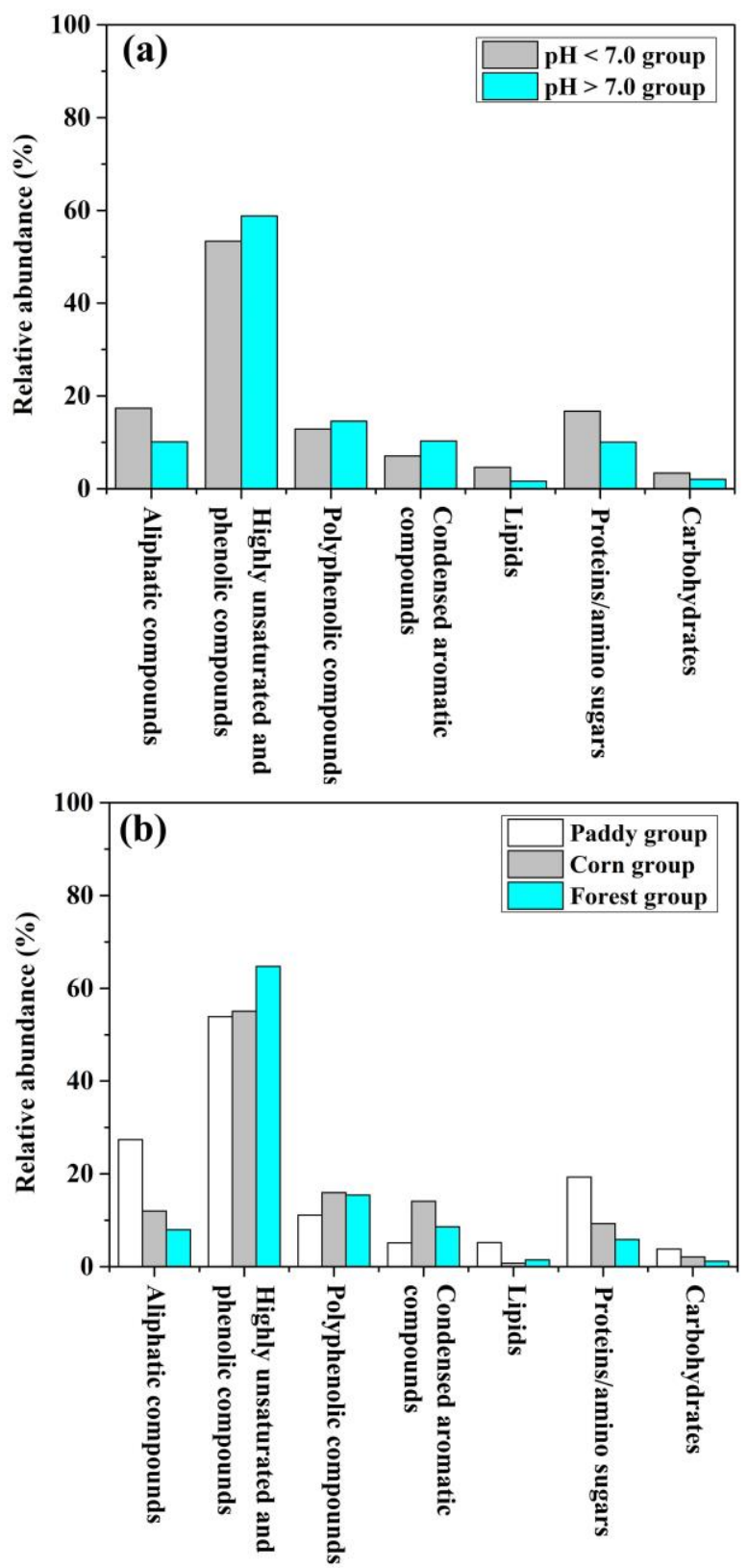

54 Figure S4. Mean values of the relative abundance of major organic compounds for (a) $\mathrm{pH}$ groups $(\mathrm{pH}>7.0$ 55 group, $\mathrm{n}=21 ; \mathrm{pH}<7.0$ group, $\mathrm{n}=12$ ) and (b) vegetation groups (paddy group, $\mathrm{n}=8$; corn group, $\mathrm{n}=4$; 56 forest group, $\mathrm{n}=2$ ). Major DOM compounds included aliphatic compounds, highly unsaturated and phenolic 57 compounds, polyphenolic compounds, condensed aromatic compounds, lipids, proteins/amino sugars, and 58 carbohydrates. 


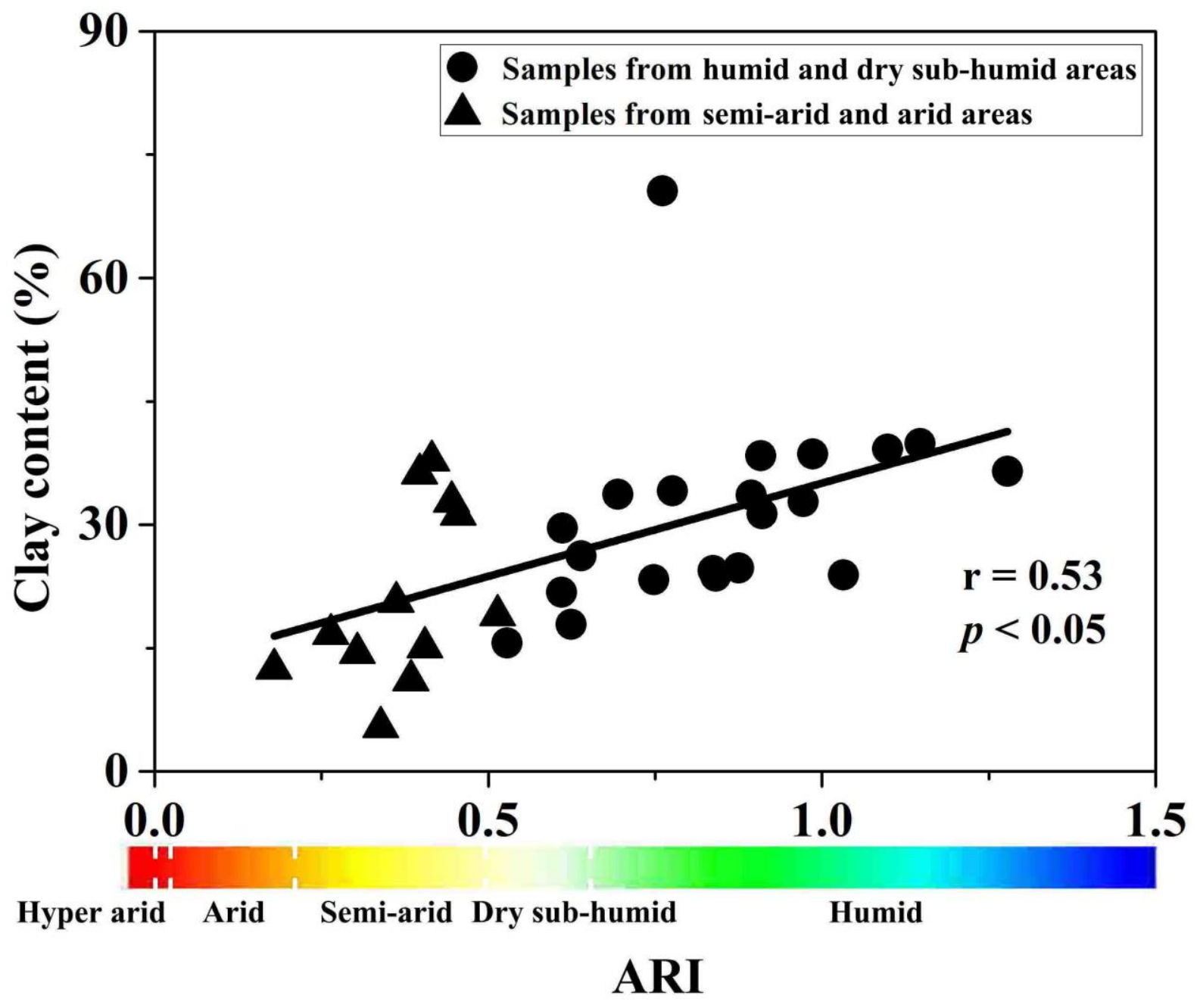

Figure S5. Pearson correlations between ARI and clay content $(n=33)$. Color bar represents the generalized 61 climate classification based on ARI values. Hyper arid, 0-0.03; arid, 0.03-0.2; semi-arid, 0.2-0.5; dry sub62 humid, 0.5-0.65; humid, > 0.65. ARI, aridity index. 

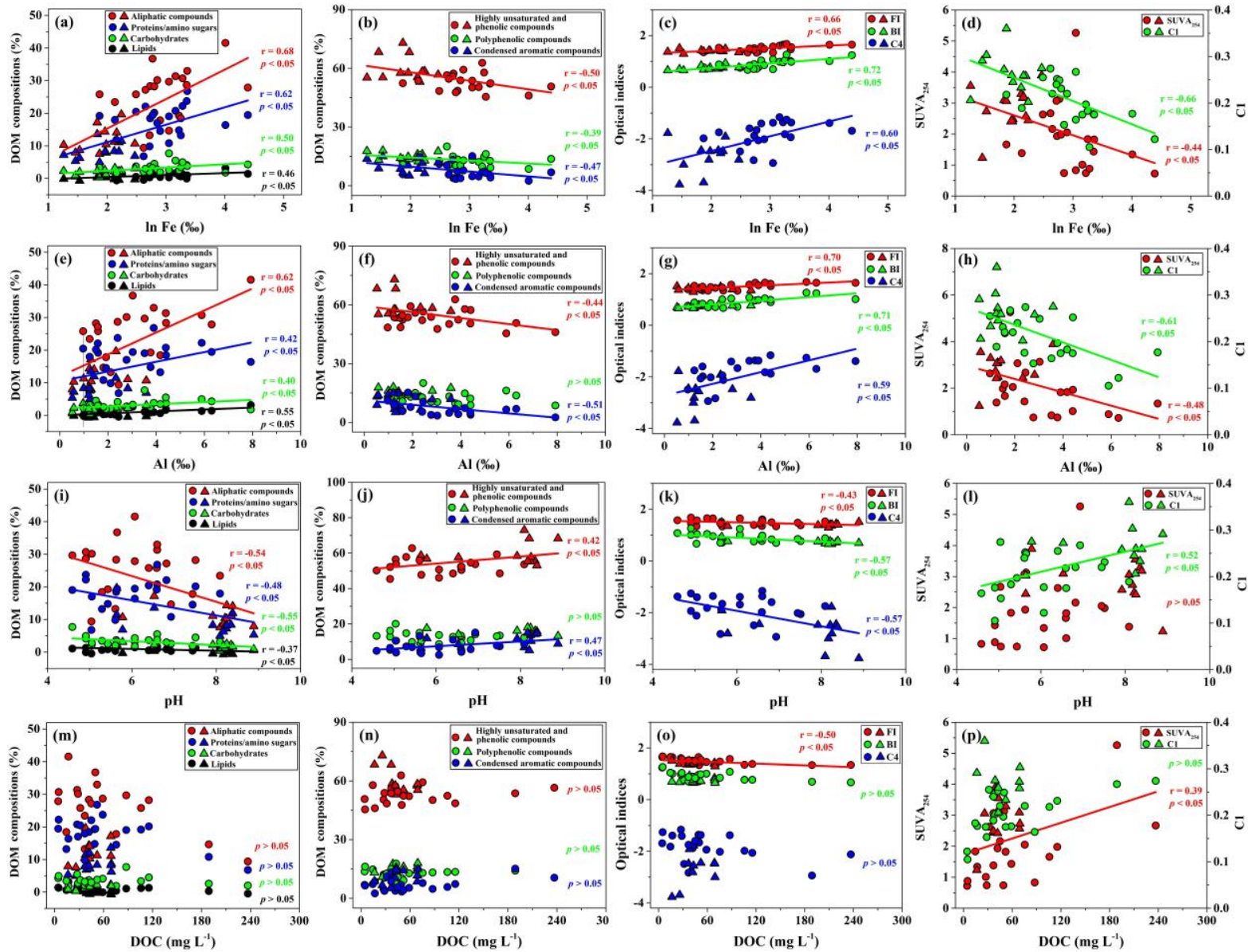

Figure S6. (a)-(d) Correlations between Fe oxide content and DOM composition or optical indices. (e)-(h) Correlations between $\mathrm{Al}$ oxide content and DOM composition or optical indices. (i)-(l) Correlations between $\mathrm{pH}$ and DOM composition or optical indices. (m)-(p) Correlations between DOC and DOM composition or optical indices. Symbols are experimental data and solid lines are linear regression. Filled triangles represent samples from semi-arid and arid areas, while filled circles represent samples from humid and dry subhumid areas. Parameters $r$ and $p$ represent Pearson correlation coefficient and significance, respectively, with the exception of BI vs environmental variables (Spearman correlations were used due to non-normally distributed data even after natural-log transformation). The data of lipids and $\mathrm{C} 4$ were treated with natural$\log$ transformation. Note that the linear correlation between DOC and polyphenolic compounds was 


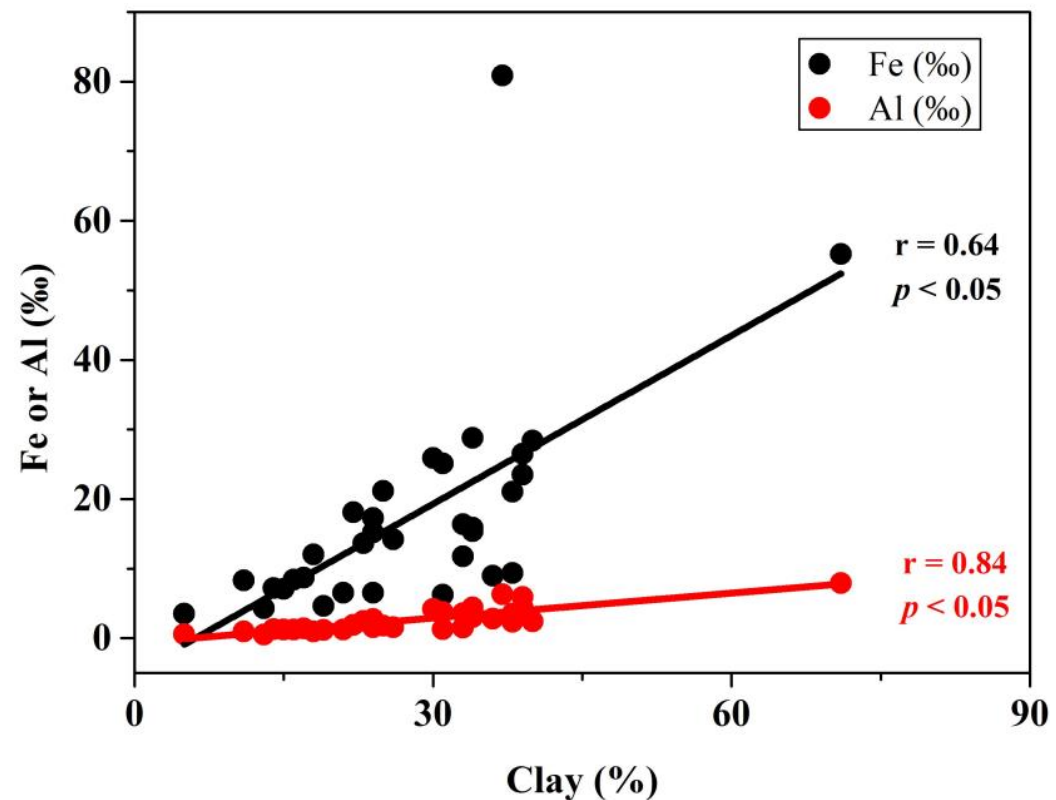

Figure S7. Correlations between clay contents and $\mathrm{Fe}$ or $\mathrm{Al}$ oxide contents for all soil samples. 
(a) Fe
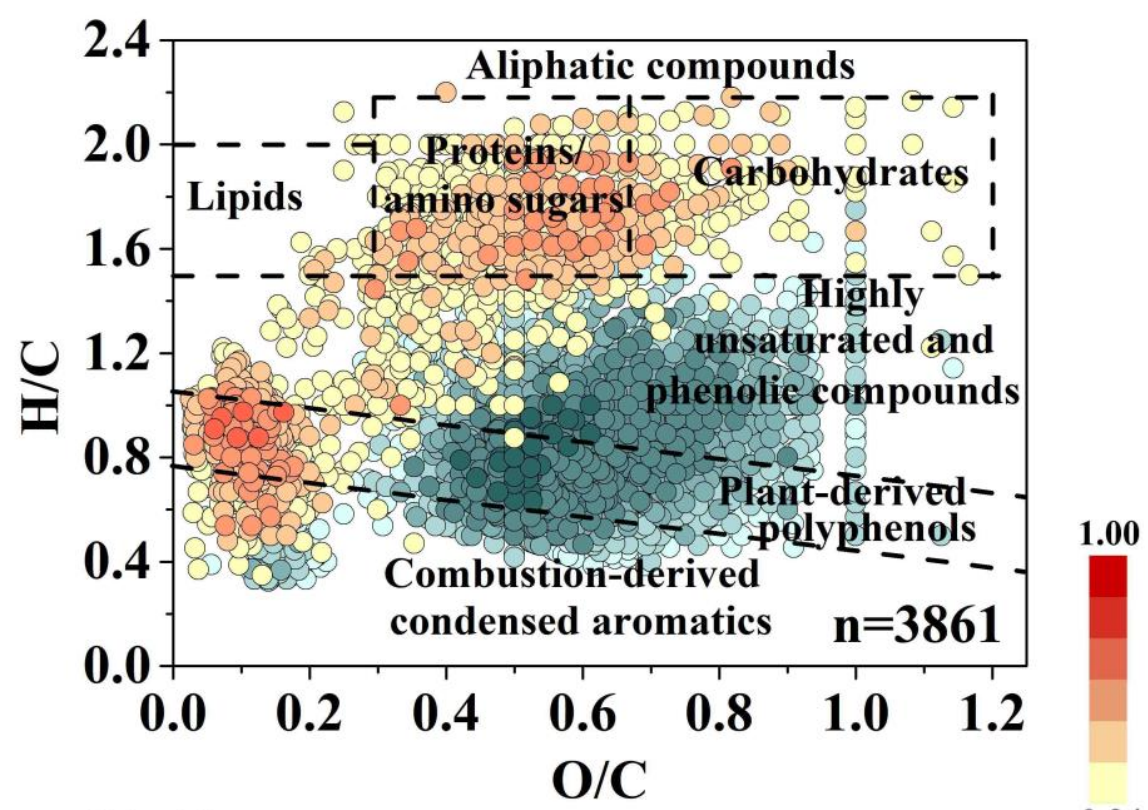

(b) Al

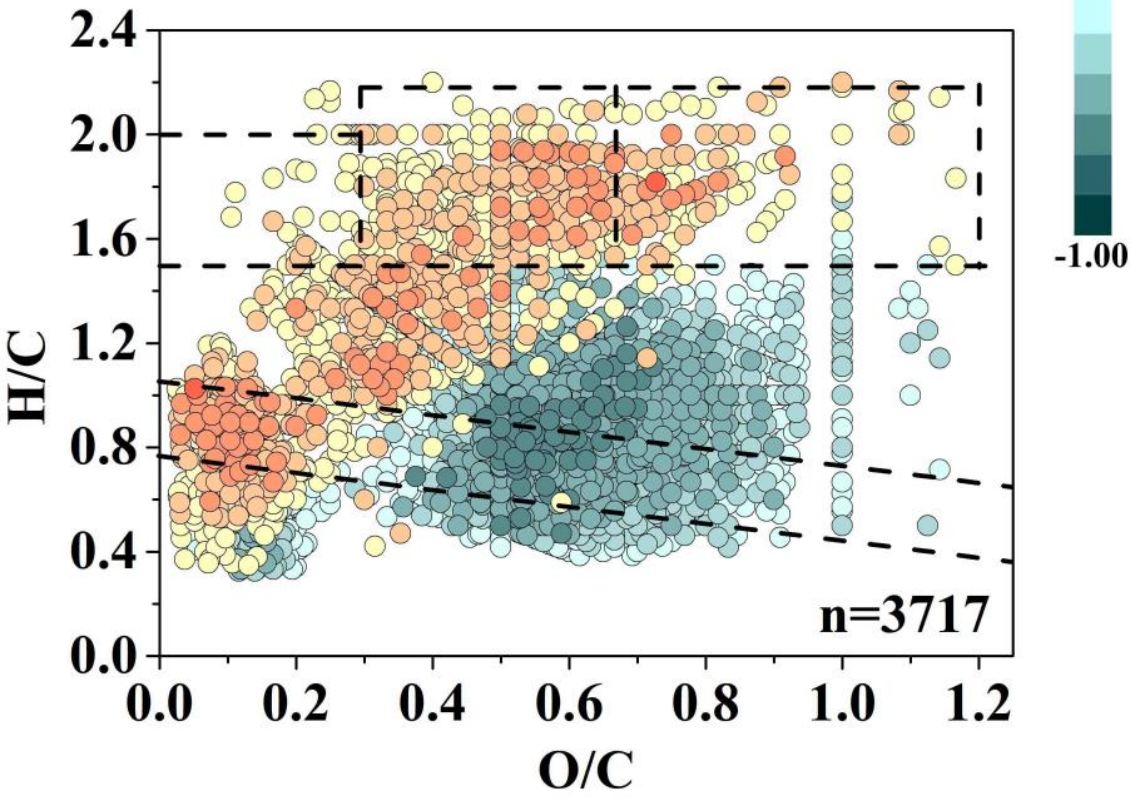

Figure S8. Associations between individual molecules and environmental variables. Significant Spearman correlations $(p<0.05)$ of individual molecules with (a) Fe oxides and (b) Al oxides are presented. Color bar indicates direction and strength of the correlation (red, positive; cyan, negative). Circles indicate DOM molecules. Lines separating compound groups include combustion-derived condensed aromatics (AI >0.66), vascular plant-derived polyphenols $(0.66 \geq \mathrm{AI}>0.5)$, highly unsaturated and phenolic compounds $(\mathrm{AI} \leq 0.5$ and $\mathrm{H} / \mathrm{C}<1.5)$, and aliphatic compounds $(2.2 \geq \mathrm{H} / \mathrm{C} \geq 1.5)$. Aliphatic compounds were divided into three components, including lipids (H/C: 1.5-2.0; O/C: 0-0.3), proteins/amino sugars (H/C: 1.5-2.2; O/C: 0.3-0.67), and carbohydrates (H/C: $1.5-2.2 ; \mathrm{O} / \mathrm{C}: 0.67-1.2)$. 


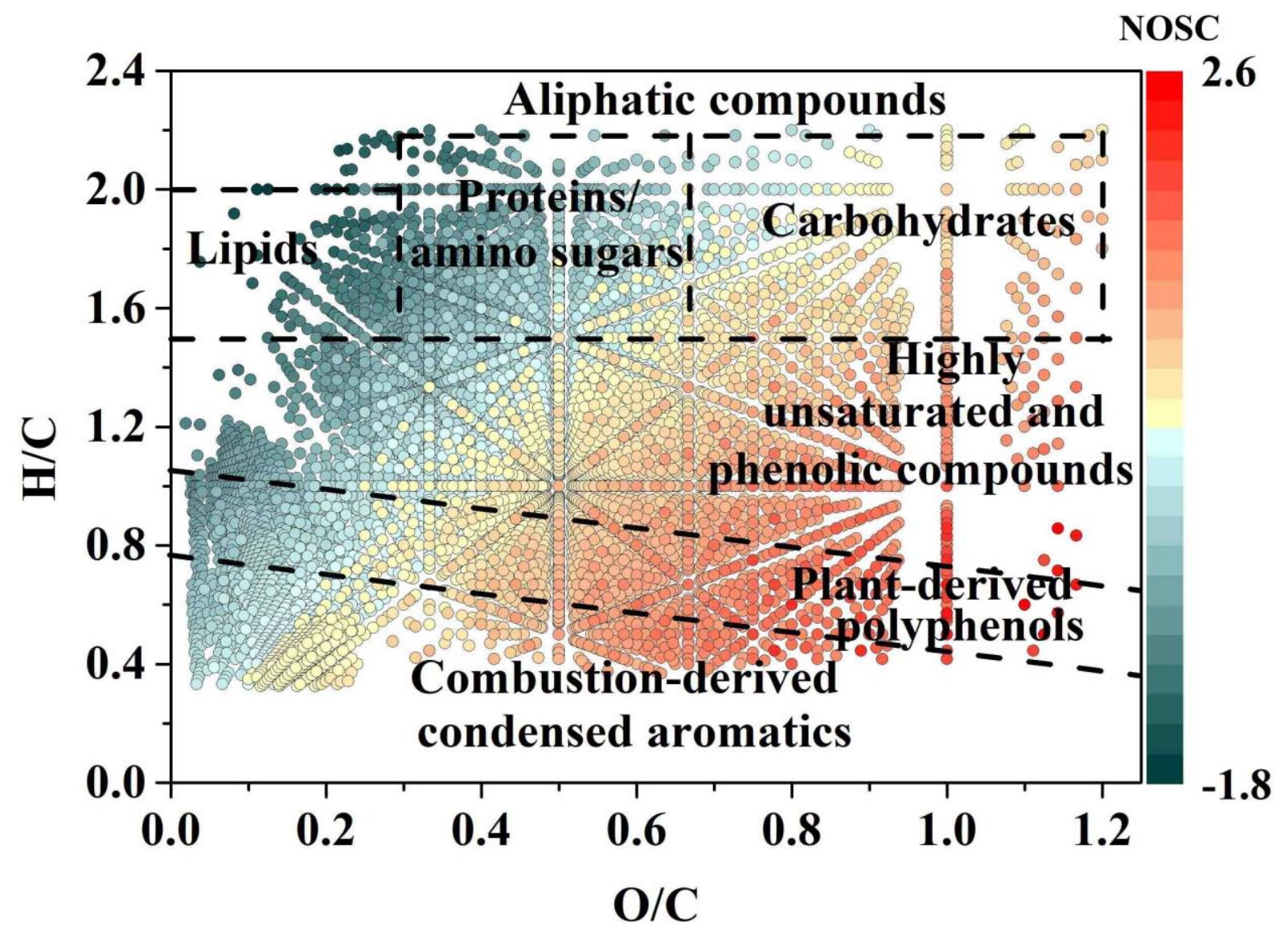

Figure S9. The VK diagram of all DOM molecules based on NOSC values. Lines separating compound groups include combustion-derived condensed aromatics (AI $>0.66)$, vascular plant-derived polyphenols $(0.66 \geq \mathrm{AI}>0.5)$, highly unsaturated and phenolic compounds $(\mathrm{AI} \leq 0.5$ and $\mathrm{H} / \mathrm{C}<1.5)$, and aliphatic compounds $(2.2 \geq \mathrm{H} / \mathrm{C} \geq 1.5)$. Aliphatic compounds were divided into three components, including lipids (H/C: 1.5-2.0; O/C: 0-0.3), proteins/amino sugars $(\mathrm{H} / \mathrm{C}: 1.5-2.2 ; \mathrm{O} / \mathrm{C}: 0.3-0.67)$, and carbohydrates $(\mathrm{H} / \mathrm{C}$ : $1.5-2.2 ; \mathrm{O} / \mathrm{C}: 0.67-1.2)$. 

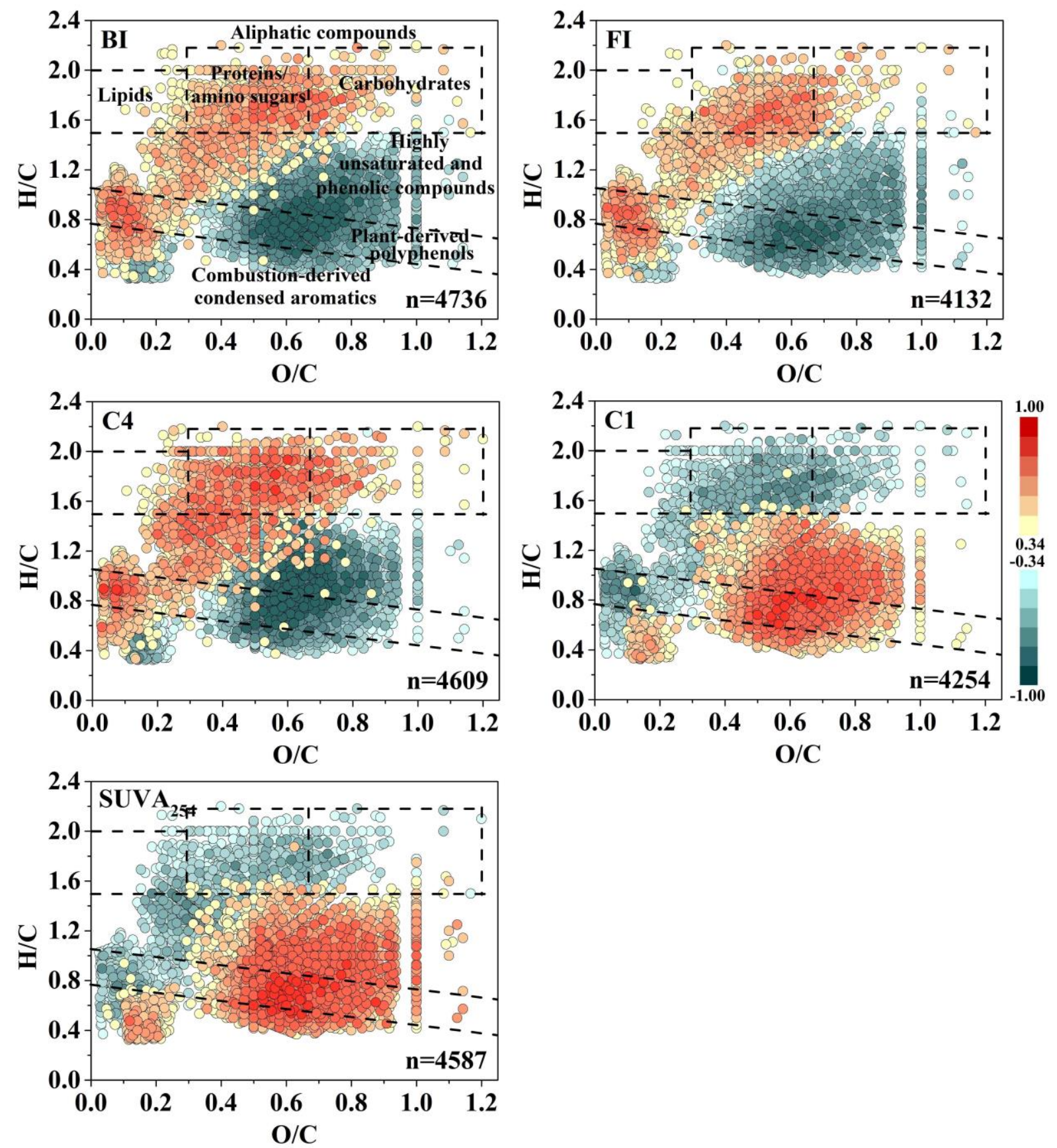

Figure S10. Associations between individual molecules and optical indices. Significant Spearman correlations $(p<0.05)$ are presented. Color bar indicates direction and strength of the correlation (red, positive; cyan, negative). Circles indicate DOM molecules. Lines separating compound groups include combustion-derived condensed aromatic (AI $>0.66$ ), vascular plant-derived polyphenols $(0.66 \geq \mathrm{AI}>0.5)$, highly unsaturated and phenolic compounds $(\mathrm{AI} \leq 0.5$ and $\mathrm{H} / \mathrm{C}<1.5)$, and aliphatic compounds $(2.2 \geq \mathrm{H} / \mathrm{C}$ 